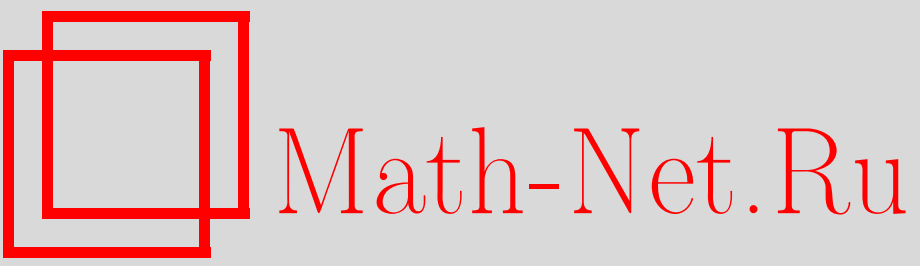

М. Д. Миссаров, РГ-инвариантные кривые в фермионной иерархической модели, ТМФ, 1998, том 114, номер 3, 323-336

DOI: https://doi.org/10.4213/tmf843

Использование Общероссийского математического портала Math-Net.Ru подразумевает, что вы прочитали и согласны с пользовательским соглашением

http://www.mathnet.ru/rus/agreement

Параметры загрузки:

IP : 35.173 .219 .149

26 апреля 2023 г., 16:28:37 
ТЕОРЕТИЧЕСКАЯ

И МАТЕМАТИЧЕСКАЯ

ФИЗИКА

Том 114, № 3

март, 1998

(C) 1998 г.

М. Д. Миссаров*

\section{РГ-ИНВАРИАНТНЫЕ КРИВЫЕ В ФЕРМИОННОЙ ИЕРАРХИЧЕСКОЙ МОДЕЛИ}

Изучается преобразование ренормализационной группы в фермионной иерархической модели, которое задается рациональным отображением в плоскости констант связи. Построены части устойчивых инвариантных кривых для всех неподвижных точек этого преобразования. Эти кривые являются графиками гладких функций, для которых даны верхняя и нижняя оценки.

\section{1. ВВЕДЕНИЕ}

В работе [1] был предложен фермионньй вариант иерархической модели Дайсона. Бозонная иерархическая модель была глубоко исследована в ряде работ [2-5] методами ренормализационной группы (РГ). Строгое обоснование положений вильсоновской картины критических явлений наиболее детально было проведено именно в рамках иерархических моделей. Действие РГ, как правило, явно не вычисляется в моделях на обычной решетке. В иерархической модели Дайсона оно сводится к нелинейному интегральному оператору, действующему на плотность распределения единичного спина. В фермионной иерархической модели действие РГ вычисляется явно и, более того, задается рациональным преобразованием в пространстве констант связи.

Напомним основные определения. Иерархическая решетка задана как множество натуральных чисел $N$ с иерархическим расстоянием $d(i, j), i, j \in N$, где $d(i, j)=n^{s(i, j)}$, если $i \neq j ; s(i, j)=\min \left\{s:\right.$ сушествует $k$ такое, что $\left.i \in V_{k, s}, j \in V_{k, s}\right\} ; V_{k, s}=\{j: j \in N$, $\left.(k-1) n^{s}<j \leq k n^{s}\right\} ; n$-некоторое фиксированное натуральное число. В узлах этой решетки находятся четырехкомпонентные спины $\psi^{*}(i)=\left(\bar{\psi}_{1}(i), \psi_{1}(i), \bar{\psi}_{2}(i), \psi_{2}(i)\right)$, компоненты которых являются образующими алгебры Грассмана. Модель задана как гиббсовское состояние на подалгебре, порожденной спинами, находящимися в узлах объема $\Lambda_{N} \equiv V_{1, N}$, где гауссовская часть задана гамильтонианом гауссовского состояния с корреляционной функцией

$$
\begin{aligned}
\left\langle\bar{\psi}_{k}(i) \psi_{l}(j)\right\rangle & =\delta_{k l}\left(\delta_{i j} \frac{1-n^{-1}}{1-n^{1-\alpha}}+\left(1-\delta_{i j}\right) d^{\alpha-2}(i, j)\right), \\
\left\langle\bar{\psi}_{k}(i) \bar{\psi}_{l}(j)\right\rangle & =\left\langle\psi_{k}(i) \psi_{l}(j)\right\rangle=0
\end{aligned}
$$

\footnotetext{
* Казанский государственный университет, Казань, Россия
} 
а взаимодействие задано потенциалом

$$
r\left(\bar{\psi}_{1}(i) \psi_{1}(i)+\bar{\psi}_{2}(i) \psi_{2}(i)\right)+g\left(\bar{\psi}_{1}(i) \psi_{1}(i) \bar{\psi}_{2}(i) \psi_{2}(i)\right)
$$

РГ-преобразование определяется по формуле

$$
r_{\alpha} \psi^{*}(i)=n^{-\alpha / 2} \sum_{j \in V_{i, 1}} \psi^{*}(j)
$$

В пространстве констант связи $(r, g)$ РГ-преобразование действует как рациональное отображение

$$
r^{\prime}=n^{\alpha-1}\left(\frac{(r+1)^{2}-g}{(r+1)^{2}-g / n}(r+1)-1\right), \quad g^{\prime}=n^{2 \alpha-3}\left(\frac{(r+1)^{2}-g}{(r+1)^{2}-g / n}\right)^{2} g
$$

которое имеет нетривиальные ветви неподвижных точек $(\mathrm{HT})\left(r_{ \pm}, g_{ \pm}\right)$, где

$$
r_{ \pm}=\frac{ \pm \sqrt{n}-n^{\alpha-1}}{1 \mp \sqrt{n}}, \quad g_{ \pm}=g_{ \pm}\left(r_{ \pm}\right), \quad g_{ \pm}(r)=\frac{r(1+r)^{2}}{1+r \pm 1 / \sqrt{n}}
$$

Отметим, что "плюсовая" ветвь $\left(r_{+}, g_{+}\right)$бифурцирует от тривиальной гауссовской ветви $r \equiv 0, g \equiv 0$ в точке $\alpha=3 / 2$ (подробнее см. [1]).

В этой работе мы дадим частичное описание устойчивых инвариантных кривых. Будет показано, что при $\alpha>3 / 2$ компонента связности устойчивой РГ-инвариантной кривой, проходящей через плюсовую (минусовую) НТ, задается графикком гладкой монотонно возрастающей (убывающей) функции $g=h_{+}(r), r>0\left(g=h_{-}(r), r<-1\right)$. При этом $h_{+}(r) \rightarrow 0$ при $r \rightarrow 0$ и $h_{-}(r) \rightarrow 0$ при $r \rightarrow-1, h_{+}(r) \rightarrow+\infty$ при $r \rightarrow+\infty$ и $h_{-}(r) \rightarrow+\infty$ при $r \rightarrow-\infty$. Будут даны верхние и нижние оценки для этих функций. При $1<\alpha \leq 3 / 2$ эти результаты также остаются справедливыми, но кривая $g=$ $h_{+}(r)$ уже будет являться устойчивой РГ-инвариантной кривой для нулевой НТ $(r=0$, $g=0)$. Кроме того, мы докажем, что нижняя полуплоскость $g<0$ при $\alpha \geq 3 / 2$ делится на две части некоторой РГ-инвариантной кривой $\gamma_{3}$, связывающей нулевую НТ и бесконечно удаленную НТ (которой на самом деле соответствует распределение грассманова спина, отвечающего $\delta$-функции). Эта кривая определяется условием принадлежности области, ограниченной по $r$.

Все эти результаты были анонсированы в работе [6], в которой исследовалась динамика РГ в областях справа от кривой $g=h_{+}(r), r>0$, и слева от кривой $g=h_{-}(r)$, $r<-1$. Было показано, что эти области РГ-инвариантны, и найдена асимптотика итераций РГ в этих областях. Доказано, что в нижней полуплоскости точки, лежашие справа и слева от $\gamma_{3}$, РГ-инвариантны. Была также найдена асимптотика этих итераций. Эти результаты были использованы при доказательстве сушествования термодинамического предела.

Поведение РГ в области между кривыми $\gamma_{1}$ и $\gamma_{2}$ более сложно. Компютерные эксперименты показывают, что устойчивые инвариантные кривые для нетривиальных НТ 
сложным образом размешаются внутри этой области. При $\alpha<3 / 2$ плюсовая НТ переходит в нижнюю полуплоскость, и начиная с некоторого $\alpha$ она становится неустойчивым фокусом.

Устойчивые РГ-инвариантные кривые можно рассматривать как зоны притяжения нетривиальных (не гауссовских) предельных законов для фермионных иерархических полей. В терминах статистической физики они называются критическими кривыми и являются ключевым элементом в картине критических явлений. Критическое поведение в фермионной иерархической модели будет описано в следующей статье.

\section{2. ИНВАРИАНТНЫЕ КРИВЫЕ}

Мы сохраняем обозначения работы [6]. Пусть

$$
\begin{aligned}
g_{1}^{ \pm}(r) & =\frac{(r+1)(n \mp \sqrt{n})-n+n^{2-\alpha}}{(r+1)(n \mp \sqrt{n})-1+n^{1-\alpha}}(r+1)^{2} \\
g_{4}(r) & =\frac{r\left(1-n^{\alpha-2}\right)}{r\left(1-n^{\alpha-2}\right)+1-n^{-1}}(r+1)^{2} .
\end{aligned}
$$

Заметим, что кривые $g_{ \pm}(r), g_{1}^{ \pm}(r)$ проходят через плюсовую и минусовую НТ, соответственно.

Пусть

$$
\begin{aligned}
G_{1}= & \left\{(r, g): 0 \leq r \leq r_{+}, \max \left\{0, g_{4}(r), g_{1}^{+}(r)\right\} \leq g \leq g_{+}(r)\right\} \cup \\
& \cup\left\{(r, g): \max \left\{0, r_{+}\right\} \leq r, g_{+}(r) \leq g \leq g_{1}^{+}(r)\right\}, \\
G_{2}= & \left\{(r, g): r \leq r_{-}, g_{1}^{-}(r) \leq g \leq g_{-}(r)\right\} \cup \\
& \cup\left\{(r, g): r_{-}<r<-1, g_{-}(r)<g<g_{1}^{-}(r)\right\} .
\end{aligned}
$$

Справедлива следуюшая теорема:

Tеорема 1. Существует гладкая возрастающая функиия $g=h_{+}(r ; \alpha), \quad 0 \leq$ $r<\infty$, график которой лежит в области $G_{1}$ и является частью $\gamma_{1}$ устойчивой РГ-инвариантной кривой для плюсовой $Н T$ при $\alpha>3 / 2$ и для тривиальной $Н$ Т при $3 / 2 \geq \alpha>1$. Существует гладкая убиввающая функиия $g=h_{-}(r ; \alpha),-\infty<$ $r<-1$, график которой лежит в области $G_{2}$ и является частью $\gamma_{2}$ устойчивой РГ-инвариантной кривой для минусовой $Н T$ при $\alpha>1$. Кроме того, все точки из множеств $G_{i} \backslash \gamma_{i}$ за конечное число итераций РГ выходят из областей $G_{i}$, $i=1,2$. В частности, точки из $G_{1}\left(G_{2}\right)$, лежсащие справа (слева) от $\gamma_{1}\left(\gamma_{2}\right)$, выходят в область, лежащую справа (слева) от $G_{1}\left(G_{2}\right)$.

Для доказательства теоремы нам понадобится несколько лемм. Мы будем в дальнейшем использовать обозначения

$$
\beta=\beta(r, g)=\frac{g}{(r+1)^{2}}, \quad \sigma=\sigma(r, g)=\frac{1-\beta(r, g)}{1-\beta(r, g) / n} .
$$


ЛЕмма 1. Пусть $\alpha>1$ и точки $\left(r_{1}, g_{1}\right),\left(r_{2}, g_{2}\right)$ удовлетворяют условиям

$$
r_{1} \leq r_{2}, \quad 1>\beta_{1} \geq \beta_{2}>0, \quad r_{i}>-1, \quad r_{i}^{\prime}>-1, \quad i=1,2,
$$

где $\beta_{i}=\beta\left(r_{i}, g_{i}\right), \quad i=1,2$. Тогда $r_{1}^{\prime} \leq r_{2}^{\prime} u \beta_{1}^{\prime} \geq \beta_{2}^{\prime}$.

ЛЕмма 2. Пусть $\alpha>1$ и точки $\left(r_{1}, g_{1}\right),\left(r_{2}, g_{2}\right)$ удовлетворяют условиям

$$
g_{1} \geq g_{2}, \quad n>\beta_{1} \geq \beta_{2}>1, \quad r_{i}<-1, \quad r_{i}^{\prime}<-1, \quad i=1,2 .
$$

Тогда $g_{1}^{\prime} \geq g_{2}^{\prime}$ и $\beta_{1}^{\prime} \geq \beta_{2}^{\prime}$.

ДокАЗАТЕЛЬСТво ЛЕмм 1 и 2. Легко видеть, что если $n>\beta_{1} \geq \beta_{2}$, то $\sigma_{1} \leq \sigma_{2}$. При этом $\sigma(\beta)>0$, если $1>\beta>0$, и $\sigma(\beta)<0$, если $n>\beta>1$. Так как $g^{\prime}=n^{2 \alpha-3} \sigma^{2} g$, то при условиях (1) имеет место неравенство $g_{1}^{\prime} \geq g_{2}^{\prime}$. Докажем, что любое из условий (1), (2) влечет за собой неравенство

$$
\left|r_{1}^{\prime}+1\right| \leq\left|r_{2}^{\prime}+1\right|
$$

В силу того что

$$
r_{i}^{\prime}+1=n^{\alpha-1} \sigma_{i}\left(r_{i}+1\right)-n^{\alpha-1}+1, \quad i=1,2,
$$

неравенство (3) равносильно неравенству

$$
\sigma_{1}\left(r_{1}+1\right) \leq \sigma_{2}\left(r_{2}+1\right)
$$

если $r_{i}^{\prime}>-1, i=1,2$, и неравенству

$$
\sigma_{1}\left(r_{1}+1\right) \geq \sigma_{2}\left(r_{2}+1\right)
$$

если $r_{i}^{\prime}<-1$. При выполнении условий (1) справедливость неравенства (4) очевидна. Справедливость неравенства (5) при выполнении условий (2) следует из того, что

$$
\sigma^{2}(r+1)^{2}=\left(\frac{1-\beta}{1-\beta / n}\right)^{2} \frac{g}{\beta},
$$

где последняя функция возрастает по $\beta$ при фиксированном $g>0$ и $n>\beta>1$. Заметим, что во всех рассматриваемых случаях

$$
r_{i}^{\prime}>-n^{\alpha-1}, \quad i=1,2 \text {, }
$$

т.к. $r^{\prime}+n^{\alpha-1}=n^{\alpha-1} \sigma(r+1)$. Для завершения доказательств лемм 1 и 2 осталось доказать неравенство $\beta_{1}^{\prime} \geq \beta_{2}^{\prime}$. Оно вытекает из неравенства (3) и формул

$$
\beta_{i}^{\prime}=\frac{1}{n} \beta_{i}\left(1+\frac{n^{\alpha-1}-1}{r_{i}^{\prime}+1}\right)^{2}, \quad i=1,2 .
$$


Лемма 3. Парабола $g(r)=\beta(r+1)^{2}, \beta=$ const, за одну итерацию РГ переходит в параболу $g^{\prime}\left(r^{\prime}\right)=\beta\left(r^{\prime}+n^{\alpha-1}\right)^{2} / n$, где $r^{\prime}$ линейно зависит от $r, r^{\prime}=k r+b, k>0$ при $\beta<1$ и $\beta>n, \quad k<0$ при $1<\beta<n$. Точки пересечения парабол $g(r)$ и $g^{\prime}(r)$ лежсат на прямых $r=r_{ \pm}$.

Утверждение леммы проверяется простым вычислением.

Заметим также, что кривые вида $g(r)=\beta(r)(r+1)^{2}$, где функция $\beta(r)=(r+a) /(r+b)$ такова, что $\beta\left(r_{+}\right)=\beta_{+}$(или $\left.\beta\left(r_{-}\right)=\beta_{-}\right)$, преобразованием РГ переводятся в кривую

$$
g^{\prime}\left(r^{\prime}\right)=\beta^{\prime}\left(r^{\prime}\right)\left(r^{\prime}+n^{\alpha-1}\right)^{2}, \quad \text { где } \quad \beta^{\prime}\left(r^{\prime}\right)=\frac{r^{\prime}+a^{\prime}}{r^{\prime}+b^{\prime}}
$$

причем $\beta^{\prime}\left(r_{ \pm}\right)=\beta_{ \pm} / n$ (или $\left.\beta^{\prime}\left(r_{-}\right)=\beta_{-} / n\right)$ и $r^{\prime}$ также дробно-линейно зависит от $r$. Здесь

$$
\beta_{ \pm}=\beta\left(r_{ \pm}, g_{ \pm}\right)=\frac{n^{\alpha-1 / 2} \mp n}{n^{\alpha-1 / 2} \mp 1} .
$$

Кривые $g=g_{1}^{ \pm}(r)$ и $g=g^{ \pm}(r)$ принадлежат как раз этому классу кривых.

Лемма 4. Имеет место равенство

$$
R\left(r, g_{1}^{ \pm}(r)\right)=\left(r_{ \pm}, \frac{g_{1}^{ \pm}(r)}{(1+r)^{2}}\left(1+r_{ \pm}\right)^{2}\right)
$$

В частности, часть кривой $g=g_{1}^{+}(r), r \geq r_{+}$, отображается на отрезок $r=r_{+}$, $g_{+} \leq g<\left(1+r_{+}\right)^{2}$, а часть кривой $g=g_{1}^{+}(r), \quad 0<r<r_{+}$, отображается на отрезок $r=r_{+}, \quad 0<g<g_{+}$. Соответственно часть кривой $g=g_{1}^{-}(r), \quad r \leq r_{-}$, отображсатся на отрезок $r=r_{-}, \quad\left(r_{-}+1\right)^{2}<g \leq g_{-}$, а часть $g=g_{1}^{-}(r), r_{-}<$ $r<-1,-$ на отрезок $r=r_{-}, g_{-}<g<n\left(r_{-}+1\right)^{2}$.

ДокАЗАТЕЛЬСТво. Прямым вычислением можно проверить, что

$$
\begin{aligned}
\frac{1}{\beta^{\prime}}-\frac{1}{\beta}= & \frac{\left(r^{\prime}(r, g)+1\right)^{2}}{g^{\prime}(r, g)}-\frac{(r+1)^{2}}{g}= \\
= & \frac{n-1}{g\left(g-(r+1)^{2}\right)^{2}}\left(r+1-\frac{1-n^{1-\alpha}}{n-\sqrt{n}}\right) \times \\
& \times\left(r+1-\frac{1-n^{1-\alpha}}{n+\sqrt{n}}\right)\left(g-g_{1}^{+}(r)\right)\left(g-g_{1}^{-}(r)\right) .
\end{aligned}
$$

Таким образом, кривые $g=g_{1}^{+}(r)$ и $g=g_{1}^{-}(r)$ характеризуются тем свойством, что для них сохраняется значение $\beta$ : $\beta=\beta^{\prime}$. Этот факт вместе с леммой 3 показывает, что образы кривых $g=g^{ \pm}(r)$ совпадают с прямыми $r=r_{ \pm}$. Оставшиеся утверждения леммы легко проверяются.

В дальнейшем мы будем использовать обозначения: $\lambda_{1}=n^{\alpha-1}, \lambda_{2}=n^{2 \alpha-3}$. 
Лемма 5. Имеет место равенство $R\left(r, g_{ \pm}(r)\right)=\left(r^{\prime}, g_{ \pm}^{\prime}\left(r^{\prime}\right)\right)$, әде

$$
g_{ \pm}^{\prime}\left(r^{\prime}\right)=\frac{r^{\prime}\left(r^{\prime}+\lambda_{1}\right)^{2}}{r^{\prime}+\lambda_{1}\left(1 \pm n^{1 / 2}\right)}
$$

$r^{\prime}$ в плюсовом и минусовом случаях вычисляется соответственно по формулам

$$
r^{\prime}= \pm \frac{\lambda_{1} r}{\left(n^{1 / 2} \mp 1\right) r+n^{1 / 2}} .
$$

В частности, часть кривой $g=g_{+}(r), r \geq r_{+}$, отображается на часть кривой $g=g_{+}^{\prime}(r), \quad r \geq r_{+}$, а часть $g=g_{+}(r), \quad 0 \leq r<r_{+},-$на часть кривой $g=g_{+}^{\prime}(r)$, $0<r<r_{+}$. Часть кривой $g=g_{-}(r), \quad r \leq r_{-}$, отображсается на часть кривой $g=g_{-}^{\prime}(r), \quad r_{-} \leq r \leq-\lambda_{1}\left(1+n^{1 / 2}\right)^{-1 / 2}$, а часть $g=g_{-}(r), \quad r_{-}<r \leq-1,-$ на часть $g=g_{-}^{\prime}(r),-\lambda_{1} \leq r<r_{-}$. При этом выполнены следующие неравенства:

$$
\begin{array}{lll}
g_{+}^{\prime}(r)<g_{+}(r) & n p u & r>\max \left(0, r_{+}\right), \\
g_{+}^{\prime}(r)>g_{+}(r) & n p u & 0<r<\max \left(0, r_{+}\right), \\
g_{-}^{\prime}(r)<g_{1}^{-}(r) & \text { npu } & r<r_{-}, \\
g_{-}^{\prime}(r)>g_{1}^{-}(r) & \text { npu } & r_{-}<r<-1 .
\end{array}
$$

ДокАЗАТЕЛЬСтво. Первая часть леммы проверяется простым вычислением. Неравенства (6), (7) следуют из разложения

$$
\begin{aligned}
g_{+}(r)-g_{+}^{\prime}(r)= & \frac{\left(n^{1 / 2}-1\right)\left(\lambda_{1}+n^{-1 / 2}\right)}{\left(r+1+n^{-1 / 2}\right)\left(r+\lambda_{1}\left(1+n^{1 / 2}\right)\right)} \times \\
& \times\left(r-r_{+}\right)\left(r+\left(1+n^{-1 / 2}\right) \frac{\lambda_{1}}{\lambda_{1}+n^{-1 / 2}}\right) .
\end{aligned}
$$

Далее, уравнение $g_{-}^{\prime}(r)=g_{1}^{-}(r)$ имеет три корня, один из которых равняется $r_{-}$. Легко видеть, что два других корня лежат правее -1 . Из того, что $r_{-}<-1, g_{1}^{-}\left(-\lambda_{1}\right)>$ $g_{-}^{\prime}\left(\lambda_{1}\right)=0$, мы получаем доказательство неравенств $(8),(9)$.

Пусть $I_{1}$ обозначает отрезок кривой, заданной в параметрической форме:

$$
I_{1}=\left\{\left(r_{1}(t), g_{1}(t)\right): t \in[0,1]\right\} .
$$

Пусть $\beta_{1}(t)=\beta\left(r_{1}(t), g_{1}(t)\right)$.

Мы будем говорить, что $I_{1}$ удовлетворяет условию А, если

$$
t_{1}<t_{2} \Rightarrow r_{1}\left(t_{2}\right) \leq r_{1}\left(t_{1}\right), \quad \beta_{1}\left(t_{1}\right) \leq \beta_{1}\left(t_{2}\right),
$$

или удовлетворяет условию Б, если

$$
t_{1}<t_{2} \Rightarrow g_{1}\left(t_{1}\right) \leq g_{1}\left(t_{2}\right), \quad \beta_{1}\left(t_{1}\right) \leq \beta_{1}\left(t_{2}\right),
$$


причем одно из двух неравенств в (10), (11) должно быть строгим.

Пусть

$$
\begin{aligned}
& G_{2}^{1}=G_{2} \cap\left\{(r, g): r_{-}<r<-1\right\}, \\
& G_{2}^{2}=G_{2} \cap\left\{(r, g): r<r_{-}\right\} .
\end{aligned}
$$

Пусть функции $F_{ \pm}(x)$ определяются формулами

$$
F_{ \pm}(x)=\frac{n}{c_{ \pm}^{2}} x\left(\frac{n-x}{x-a_{ \pm}}\right)^{2}
$$

где

$$
c_{ \pm}=1+\lambda_{1}\left(n \mp n^{1 / 2}\right), \quad a_{ \pm}=1+\frac{n-1}{c_{ \pm}} .
$$

Обозначим через $x_{ \pm}(\beta)$ корни уравнений $\beta=F_{ \pm}(x)$, удовлетворяющие условиям $0<$ $x_{+}(\beta)<1$ в плюсовом случае и $1<x_{-}(\beta)<n$ в минусовом. Позже мы покажем, что $x_{ \pm}(\beta)$ определены однозначно.

Лемма 6. Пусть $I_{1} \subset G_{2}^{1}\left(G_{2}^{2}\right)$, причем $g_{1}(0)=g_{-}\left(r_{1}(0)\right), g_{1}(1)=g_{1}^{-}\left(r_{1}(1)\right)($ coответственно $\left.g_{1}(0)=g_{1}^{-}\left(r_{1}(0)\right), g_{1}(1)=g_{-}\left(r_{1}(1)\right)\right), I_{1}$ удовлетворяет условию Б. Тогда существует такой интервал $\left[a_{1}, b_{1}\right] \subset[0,1]$, что

$$
I_{2}=\left\{\left(r_{1}^{\prime}(t), g_{1}^{\prime}(t)\right): t \in\left[a_{1}, b_{1}\right]\right\}=R I_{1} \cap G_{2}^{2}
$$

(соответственно $\left.I_{2}=R I_{1} \cap G_{2}^{1}\right)$. Здесь $\left(r^{\prime}, g^{\prime}\right)=R(r, g), R I_{1}$ - образ $I_{1}$ при отображении $R$. При этом $g_{1}^{\prime}\left(a_{1}\right)=g_{1}^{-}\left(r_{1}^{\prime}\left(a_{1}\right)\right), g_{1}^{\prime}\left(b_{1}\right)=g_{-}\left(r_{1}^{\prime}\left(b_{1}\right)\right)$ (соответственно $\left.g_{1}^{\prime}\left(a_{1}\right)=g_{-}\left(r_{1}^{\prime}\left(a_{1}\right)\right), \quad g_{1}^{\prime}\left(b_{1}\right)=g_{1}^{-}\left(r_{1}^{\prime}\left(b_{1}\right)\right)\right), I_{2}$ удовлетворяет условию Б. Если $I_{1} \subset G_{2}^{1}, m o$

$$
\beta\left(r_{1}^{\prime}\left(a_{1}\right), g_{1}^{\prime}\left(a_{1}\right)\right)>x_{-}\left(\beta_{1}(1)\right),
$$

а если $I_{1} \subset G_{2}^{2}$, mo

$$
\beta\left(r_{1}^{\prime}\left(b_{1}\right), g_{1}^{\prime}\left(b_{1}\right)\right)<x\left(\beta_{1}(0)\right)
$$

ДокАЗАТЕЛЬСТво. Пусть $I_{1} \subset G_{2}^{1}$. Так как для всех точек $(r, g) \in G_{2}^{1}, \quad 1<$ $\beta(r, g)<n$, то легко видеть, что для этих точек $r^{\prime}(r, g)<r_{-}$. В силу лемм $2,4,5$ и того, что $\beta(r, g)$ монотонно убывает по $r$ для точек кривых $g=g_{-}(r), g=g_{1}^{-}(r)$ при $r<-1$, и из условия Б следует, что $I_{2}=R I_{1} \cap G_{2}^{2}$ является связной частью кривой $R I_{1}$ и удовлетворяет условию Б. Интервал $\left[a_{1}, b_{1}\right]$ определяется как

$$
\left[a_{1}, b_{1}\right]=\left\{t \in[0,1]:\left(r^{\prime}(t), g^{\prime}(t)\right) \in I_{2}\right\}
$$

Так как кривая $I_{1}$ лежит ниже параболы с вершиной в точке $(-1,0)$, проходящей через точку $\left(r_{1}(1), g_{1}(1)\right)$, то $I_{2}$ лежит ниже параболы с вершиной в точке $\left(-\lambda_{1}, 0\right)$, проходящей через точку $\left(r_{1}^{\prime}(1), g_{1}^{\prime}(1)\right)$. В соответствии с леммой 3 последняя парабола задается уравнением $g=\beta_{1}(1)\left(r+\lambda_{1}\right)^{2} / n$. Обозначим координаты точки пересечения этой параболы с кривой $g=g_{1}^{-}(r)$ через $\left(r_{0}, g_{0}\right)$. 
Делая замену переменных

$$
x=\frac{(r+1)(n+\sqrt{n})-n\left(1-n^{1-\alpha}\right)}{(r+1)(n+\sqrt{n})-\left(1-n^{1-\alpha}\right)},
$$

получаем, что $\beta\left(r_{0}, g_{0}\right)$ является корнем уравнения $\beta_{1}(1)=F_{-}(x)$ таким, что $1<x<n$. Из того, что точки $\left(r_{0}, g_{0}\right)$ и $\left(r_{1}^{\prime}\left(a_{1}\right), g_{1}^{\prime}\left(a_{1}\right)\right)$ лежат на кривой $g=g_{1}^{-}(r)$ и $r_{0}<r_{1}^{\prime}\left(a_{1}\right)$, следует вторая часть леммы.

Случай, когда $I_{1} \subset G_{2}^{2}$, рассматривается аналогично.

Рассмотрим ситуацию в правом квадранте верхней полуплоскости, в котором находится плюсовая НТ при $\alpha \geq 3 / 2$ (при $\alpha=3 / 2$ плюсовая НТ совпадает с тривиальной НТ). Пусть $G_{1}^{1}=G_{1} \cap\left\{(r, g): r>r_{+}\right\}, G_{1}^{2}=G_{1} \cap\left\{(r, g): 0<r<r_{+}\right\}$.

Лемма 7. Пусть $I_{1} \subset G_{1}^{1}\left(G_{1}^{2}\right)$, причем

$$
g_{1}(0)=g_{+}\left(r_{1}(0)\right), \quad g_{1}(1)=g_{1}^{+}\left(r_{1}(1)\right)
$$

(соответственно $\left.g_{1}(0)=g_{1}^{+}\left(r_{1}(0)\right), g_{1}(1)=g_{+}\left(r_{1}(1)\right)\right), I_{1}$ удовлетворяет условию А. Тогда существует такой интервал $\left[a_{1}, b_{1}\right] \subset[0,1]$, что

$$
I_{2}=\left\{\left(r_{1}^{\prime}(t), g_{1}^{\prime}(t)\right): t \in\left[a_{1}, b_{1}\right]\right\}=R I_{1} \cap G_{2}^{1}
$$

(соответственно $\left.I_{2}=R I_{1} \cap G_{2}^{1}\right) . \quad$ При этом $g_{1}^{\prime}\left(a_{1}\right)=g_{+}\left(r_{1}^{\prime}\left(a_{1}\right)\right), \quad g_{1}^{\prime}\left(b_{1}\right)=$ $g_{1}^{+}\left(r_{1}^{\prime}\left(b_{1}\right)\right)$ (соответственно $\left.g_{1}^{\prime}\left(a_{1}\right)=g_{1}^{+}\left(r_{1}^{\prime}\left(a_{1}\right)\right), \quad g_{1}^{\prime}\left(b_{1}\right)=g_{+}\left(r_{1}^{\prime}\left(b_{1}\right)\right)\right), I_{2}$ удовлетворяет условию А. Если $I_{1} \subset G_{1}^{1}$, то

$$
\beta\left(r_{1}^{\prime}\left(b_{1}\right), g_{1}^{\prime}\left(b_{1}\right)\right)<x_{+}\left(\beta_{1}(1)\right)
$$

если $I_{1} \subset G_{1}^{2}$, mo

$$
\beta\left(r_{1}^{\prime}\left(a_{1}\right), g_{1}^{\prime}\left(a_{1}\right)\right)>x_{+}\left(\beta_{1}(0)\right) .
$$

ДокАЗАТЕЛЬСТВо этой леммы аналогично доказательству леммы 6.

В силу леммы 3 парабола $g=\beta(r+1)^{2}, \beta<1$, при РГ-преобразовании переходит в параболу $g=\beta\left(r+\lambda_{1}\right)^{2} / n$ и пересечение этих парабол происходит при $r=r_{+}$. Если конец отрезка $I_{1}\left(r_{1}(1), g_{1}(1)\right)$ лежит на кривой $g=g_{1}^{+}(r)$, а сам отрезок находится в области $G_{1}^{1}$, то после итерации РГ $I_{1}$ растянется так, что $\left(r_{1}^{\prime}(1), g_{1}^{\prime}(1)\right)$ будет лежать на прямой $r=r_{+}$, а $\left(r_{1}^{\prime}(0), g_{1}^{\prime}(0)\right)$ - на кривой $g=r\left(r+\lambda_{1}\right)^{2}\left(r+\lambda_{1}\left(1+n^{1 / 2}\right)\right)^{-1}$ (в силу лемм 4 и 5). Так как $I_{2}=R I_{1} \cap G_{1}^{1}$ будет лежать ниже параболы

$$
g=\beta\left(r_{1}(1), g_{1}(1)\right)\left(r+\lambda_{1}\right)^{2} / n
$$

то для любой точки $(r, g) \in I_{2}$ будем иметь $\beta(r, g)<\beta(\tilde{r}, \tilde{g})$, где $(\tilde{r}, \tilde{g})$ - точка пересечения параболы (16) с кривой $g=g_{1}^{+}(r)$. Нетрудно показать, что $\beta(\tilde{r}, \tilde{g})$ является корнем уравнения $\beta\left(r_{1}(1), g_{1}(1)\right)=F_{+}(x)$ таким, что $0<x<1$. Тот факт, что $I_{2}$ удовлетворяет условию А, следует из леммы 1 . 
Можно показать, что если $I_{1} \subset G_{2}^{2}$ и точка $\left(r_{1}(0), g_{1}(0)\right)$ лежит не на кривой $g=$ $g_{1}^{+}(r)$, а на кривой $g=g_{4}(r)$ или на прямой $g=0$, то через конечное число $k$ итераций РГ можно добиться того, что $R^{k} I_{1} \cap G_{1}^{1}=I_{k}$ будет представлять собой криволинейный отрезок, удовлетворяюший условию леммы 7.

Рассмотрим, наконец, случай, когда $1<\alpha<3 / 2$. Здесь плюсовая НТ перейдет в нижнюю полуплоскость, зато тривиальная НТ $(0,0)$ станет устойчивой по одному направлению. В этом случае множество $G_{1}$ задается как множество $\left\{(r, g): 0 \leq r, g_{+}(r) \leq\right.$ $\left.g \leq g_{1}^{+}(r)\right\}$. Обозначим через $\gamma$ кривую, образованную участком вертикальной прямой $\left\{(r, g): r=0,0 \leq g \leq g_{1}^{+}(0)\right\}$ и кривой $g=g_{1}^{+}(r), r>0$.

ЛЕмма 8. Пусть $1<\alpha<3 / 2, \quad I_{1} \subset G_{1}, \quad$ причем $g_{1}(0)=g_{+}\left(r_{1}(0)\right)$, $\left(r_{1}(1), g_{1}(1)\right) \in \gamma, \quad I_{1}$ удовлетворяет условию А. Тогда существует интервал $\left[a_{1}, b_{1}\right] \subset[0,1]$ такой, что

$$
I_{2}=\left\{\left(r_{1}^{\prime}(t), g_{1}^{\prime}(t)\right): t \in\left[a_{1}, b_{1}\right]\right\}=R I_{1} \cap G_{1} .
$$

При этом $g_{1}^{\prime}\left(a_{1}\right)=g_{+}\left(r_{1}\left(a_{1}\right)\right), \quad\left(r_{1}^{\prime}\left(b_{1}\right), g_{1}^{\prime}\left(b_{1}\right)\right) \in \gamma, I_{2}$ удовлетворяет неравенству (12).

ДоКАЗАТЕЛЬСТВО этой леммы аналогично доказательству леммы 6. Отметим только, что отрезок $r=0,0<g<g_{1}^{+}(0)$ при одной итерации РГ перейдет в отрезок кривой

$$
g=\frac{\left(r+\lambda_{1}\right)^{2}}{r-\lambda_{1}(n-1)}, \quad r_{+}<r<0
$$

и тем самым РГ-образ кривой $\gamma$ будет лежать левее кривой $\gamma$. Далее схема доказательства совпадает со схемой доказательства леммы 6 .

Лемма 9. Уравнение

$$
x_{0}=F_{-}(x),
$$

где $1<x_{0}<n$, имеет единственное решение в области $1<x<n$. Последовательность $x_{n}$, задаваемая соотношениями

$$
x_{n-1}=F_{-}\left(x_{n}\right), \quad 1<x_{n}<n,
$$

при любом $x_{0} \in(1, n)$ сходится $\kappa \beta_{-}$.

Уравнение

$$
x_{0}=F_{+}(x),
$$

әде $0<x_{0}<1$, имеет единственное решение в области $0<x<1$. Последовательность $x_{n}$, задаваемая соотночениями

$$
x_{n-1}=F_{+}\left(x_{n}\right), \quad 0<x_{n}<1,
$$

при любом $x_{0} \in(0,1)$ сходится $\kappa \beta_{+}$, если $\alpha>3 / 2$, ик 0 , если $1<\alpha \leq 3 / 2$. 
ДокАЗАТЕльСтво. Уравнение $x_{0}=F_{-}(x)$ имеет, вообще говоря, три различных действительных корня при $x_{0}>0$. Анализ функции $F_{-}(x)$ показывает, что она монотонно возрастает в области $\left[0, a_{-}\right)$, монотонно убывает в области $\left(a_{-}, n\right]$ и монотонно возрастает в области $(n, \infty)$. Легко заметить, что $F_{-}(1)=n$. Так как $a_{-}>1$, то в силу монотонного возрастания $F_{-}$на участке $\left[1, a_{-}\right)$мы заключаем, что интересующее нас значение корня лежит в области $\left(a_{-}, n\right)$. В этой области функция $F_{-}$имеет обратную функцию.

Пусть $F_{-}^{-1}$ обозначает отображение $F_{-}^{-1}:[1, n] \rightarrow\left[b_{1}, d_{1}\right]$, обратное к $F_{-}$на отрезке $\left[b_{1}, d_{1}\right]$. Здесь $b_{1}$ и $d_{1}$ однозначно определяются из условий $n=F_{-}\left(b_{1}\right), a_{-}<b_{1}<n$, $1=F_{-}\left(d_{1}\right), \quad a_{-}<d_{1}<n$. Разрешимость этих уравнений следует из того, что $F_{-}(x) \rightarrow \infty$ при $x \rightarrow a_{-}$, и того, что $F_{-}(n)=0$. Так как $F_{-}^{-1}$ строго монотонно убывает на $[1, n]$, то $F_{-}^{-2}$ монотонно возрастает на $[1, n]$, причем очевидно, что $F_{-}^{-2}(1)>1$, $F_{-}^{-2}(n)<n$. Покажем, что уравнение $F_{-}^{-2}(x)=x$ имеет единственное решение $x=\beta_{-}$ на интервале $[1, n]$. Эквивалентное утверждение состоит в том, что уравнение $F_{-}^{2}(x)=x$ обладает единственным решением $\beta_{-}$на отрезке $\left(a_{-}, n\right]$.

Действительно, пусть $F_{-}^{2}(x)=x$. В этом случае $F_{-}(x)$ должно быть одним из трех корней уравнения $F_{-}(y)=x(y-$ неизвестное, $x$ - параметр, $1<x<n)$. Но из сказанного вьше следует, что один из этих корней меньше 1 , а другой больше $n$. Значит, уравнение $F_{-}^{2}(x)=x$ при условии $1<F_{-}(x)<n$ сводится к уравнению $F_{-}(x)=x$, $1<x<n$, которое имеет единственное решение $x=\beta_{-}$. Из этого факта и монотонности $F_{-}^{-2}$ на $[1, n]$ следует утверждение о сходимости последовательности $x_{n}=F_{-}^{-1}\left(x_{n-1}\right)$ к $\beta_{-}$.

Сушествование и единственность решения уравнения (18) в области $0<x<1$ следует из того, что $F_{+}(x)$ непрерывна и монотонно возрастает на этом интервале и $F_{+}(0)=0$, $F_{+}(1)=n$. При $\alpha>3 / 2$ отображение $F_{+}$на $[0,1]$ имеет единственную устойчивую НТ $x=\beta_{+}$, а при $1<\alpha<3 / 2$ устойчивой становится тривиальная НТ $x=0$. Отсюда и следуют оставшиеся утверждения леммы.

ДОкаЗАтельСтво теОРемы 1. Рассмотрим случай минусовой НТ. Пусть для определенности $I_{1} \subset G_{2}^{1}$. Рассмотрим последовательность отрезков $I_{k}$, определяемых соотношением

$$
I_{k}=R I_{k-1} \cap G_{2}, \quad k=2, \ldots .
$$

Тогда последовательность $T_{k}=R^{-k+1} I_{k}$ образует последовательность замкнутых, вложенных друг в друга отрезков (подмножеств отрезка $I_{1}$ ). Таким образом, $T=$ $\bigcap_{k=1}^{\infty} T_{k}$ является замкнутым связным подмножеством отрезка $I_{1}$. Покажем, что для всех точек $(r, g) \in T$

$$
R^{k}(r, g) \rightarrow\left(r_{-}, g_{-}\right) \quad \text { при } k \rightarrow \infty .
$$

Обозначим через $x_{k}$ последовательность точек, которая определяется из соотношений

$$
x_{k-1}=F_{-}\left(x_{k}\right), \quad 1<x_{k}<n, \quad x_{0}=\beta\left(r_{1}(1), g_{1}(1)\right) .
$$

В силу леммы 6 для любой точки $(r, g) \in I_{k}$ имеем $\beta_{-}<\beta(r, g)<x_{k}$ при нечетных $k$ и $x_{k}<\beta(r, g)<\beta_{-}$для четных $k$. Если мы докажем, что $x_{k} \rightarrow \beta_{-}$при $k \rightarrow \infty$, то из 
соотношений

$$
\begin{array}{ll}
I_{k} \subset G_{2}^{1} \cap\left\{(r, g): \beta_{-}<\beta(r, g) \leq x_{k}\right\}, & k=1,3,5, \ldots, \\
I_{k} \subset G_{2}^{2} \cap\left\{(r, g): x_{k} \leq \beta(r, g)<\beta_{-}\right\}, & k=2,4,6, \ldots,
\end{array}
$$

и будет следовать (19), поскольку множества из правых частей $(21),(22)$, начиная с некоторого $k$, попадают в сколь угодно малую окрестность точки $\left(r_{-}, g_{-}\right)$.

Случай плюсовой НТ устроен еше проще. Пусть, например, $I_{1}=\left\{(r, g): r=r_{0}\right\} \cap G_{1}^{1}$ (считаем, что $r_{0}>r_{+}, \alpha \geq 3 / 2$ ). Очевидно, что отрезок $I_{1}$ удовлетворяет условию леммы 7 .

Пусть $I_{k}=R I_{k-1} \cap G_{1}^{1}, \quad k=2,3, \ldots$ Тогда последовательность отрезков $T_{k}=$ $R^{-k+1} I_{k}, \quad k=2,3, \ldots$, образует последовательность замкнутых, вложенных друг в друга отрезков (подмножеств $\left.I_{1}\right)$. Для любой точки

$$
(r, g) \in T=\bigcap_{k=1}^{\infty} T_{k}
$$

имеем $R^{k}(r, g) \rightarrow\left(r_{+}, g_{+}\right)$при $k \rightarrow \infty$.

В самом деле, согласно неравенству (14) для любой точки $(r, g) \in I_{k}$ верно, что

$$
\beta_{+}<\beta(r, g)<x_{k}, \quad k=1,2, \ldots
$$

где последовательность $x_{k}$ задается соотношением $x_{k-1}=F_{+}\left(x_{k}\right), 0<x_{k}<1, x_{0}=$ $\beta\left(r_{0}, g_{1}^{+}\left(r_{0}\right)\right)$. Вследствие того что $x_{k}$ сходится к $\beta_{+}$, мы получаем требуемое утверждение. Оставшиеся случаи рассматриваются аналогично.

Нам осталось доказать, что множество $T$ как в плюсовом, так и в минусовом случаях состоит из единственной точки.

Рассмотрим сначала случай плюсовой НТ. Пусть $T$ является отрезком: $T=\{(r, g)$ : $\left.r=r_{0}, \tilde{g}_{2}<g<\tilde{g}_{1}\right\}, r_{0}>0$. Легко видеть, что за одну итерацию РГ отрезок $T$ переходит в криволинейный отрезок $R T$ с концами $\left(r_{1}, g_{1}\right),\left(r_{2}, g_{2}\right)$, где $r_{1}<r_{2}$. Пусть

$$
\left(r_{i}^{(k)}, g_{i}^{(k)}\right)=R^{k}\left(r_{i}, g_{i}\right), \quad i=1,2 .
$$

Из определения $T$ и леммы 1 следует, что $r_{2}^{(k)}>r_{1}^{(k)}>-1,1>\beta_{1}^{(k)}>\beta_{2}^{(k)}$, где $\beta_{i}^{(k)}=$ $\beta\left(r_{i}^{(k)}, g_{i}^{(k)}\right), i=1,2$. Тогда $\sigma_{1}^{(k)}<\sigma_{2}^{(k)}$, где $\sigma_{i}^{(k)}=\left(1-\beta_{i}^{(k)}\right) /\left(1-\beta_{i}^{(k)} / n\right), i=1,2$. При $\beta \rightarrow \beta_{+}$получаем $\sigma(\beta)=(1-\beta) /(1-\beta / n) \rightarrow n^{3 / 2-\alpha}$. Поскольку $\beta_{2}^{(k)} \rightarrow \beta_{+}$, то из неравенства

$$
r_{2}^{(k+1)}-r_{1}^{(k+1)}=\lambda_{1}\left(\sigma_{2}^{(k)}\left(r_{2}^{(k)}+1\right)-\sigma_{1}^{(k)}\left(r_{1}^{(k)}+1\right)\right) \geq \lambda_{1} \sigma_{2}^{(k)}\left(r_{2}^{(k)}-r_{1}^{(k)}\right)
$$

следует, что начиная с некоторого $k$

$$
r_{2}^{(k+1)}-r_{1}^{(k+1)} \geq\left(r_{2}^{(k)}-r_{1}^{(k)}\right) .
$$


Это входит в противоречие с тем фактом, что $r_{2}^{(k)}-r_{1}^{(k)} \rightarrow 0$ при $k \rightarrow \infty$.

Рассмотрим теперь случай минусовой НТ.

Пусть $T$ является отрезком: $T=\left\{(r, g): r=r_{0}, g_{2}<g<g_{1}\right\}, r_{0}<-1$. В этом случае мы находимся в условиях леммы 2 . Поэтому $n>\beta_{1}^{(k)}>\beta_{2}^{(k)}>1, r_{i}^{(k)}<-1$, $i=1,2, g_{1}^{(k)}>g_{2}^{(k)}, k=1,2, \ldots$. Так как $\left(\sigma_{1}^{(k)}\right)^{2}>\left(\sigma_{2}^{(k)}\right)^{2}$, то мы имеем

$$
\begin{aligned}
g_{1}^{(k)}-g_{2}^{(k)} & =\lambda_{2}\left(\left(\sigma_{1}^{(k-1)}\right)^{2} g_{1}^{(k-1)}-\left(\sigma_{2}^{(k-1)}\right)^{2} g_{2}^{(k-1)}\right) \geq \\
& \geq \lambda_{2}\left(\sigma_{1}^{(k-1)}\right)^{2}\left(g_{1}^{(k-1)}-g_{2}^{(k-1)}\right) \geq \lambda_{2}^{k}\left(\sigma_{1}^{(k-1)} \sigma_{1}^{(k-2)} \ldots \sigma_{1}\right)^{2}\left(g_{1}-g_{2}\right) .
\end{aligned}
$$

Из соотношений (21), (22) следует, что $\left|\beta_{1}^{(k)}-\beta_{-}\right|<\left|x_{k}-\beta_{-}\right|, \quad k=1,2, \ldots$, где $x_{k}$ определяются соотношением (20).

Легко проверить, что в окрестности $x=\beta_{-}$отображение $F_{-}^{-1}$ является сжимающим, поэтому мы можем утверждать, что

$$
\left|\beta_{1}^{(k)}-\beta_{-}\right|<c_{1} d^{k}, \quad d<1 .
$$

Из представления

$$
\sigma_{1}^{(k)}=\frac{1-\beta_{-}}{1-\beta_{-} / n} f\left(\beta_{1}^{(k)}\right)=-\lambda_{2}^{-1 / 2} f\left(\beta_{1}^{(k)}\right),
$$

где

$$
f\left(\beta_{1}^{(k)}\right)=\frac{1-\left(\beta_{1}^{(k)}-\beta_{-}\right) /\left(1-\beta_{-}\right)}{1-\left(\beta_{1}^{(k)}-\beta_{-}\right) /\left(n\left(n-\beta_{-}\right)\right)},
$$

и из того, что бесконечное произведение $\prod_{k} f\left(\beta_{1}^{(k)}\right)$ сходится в силу (23), мы получаем

$$
g_{1}^{(k)}-g_{2}^{(k)} \geq c_{2}\left(g_{1}-g_{2}\right), \quad c_{2}>0 .
$$

Это противоречит тому, что $g_{1}^{(k)}-g_{2}^{(k)} \rightarrow 0$ при $n \rightarrow \infty$.

Перейдем к рассмотрению инвариантных кривых в нижней полуплоскости $g<0$ при $\alpha \geq 3 / 2$. Тривиальная НТ $(0,0)$ является отталкивающей при $\alpha>3 / 2$. Бесконечное множество неустойчивых инвариантных кривых, выходящих из нуля, являются устойчивыми инвариантными кривыми для бесконечно удаленной НТ, отвечаюшей потенциалу, задаваемому грассмановой $\delta$-функцией $\delta(\psi)=\bar{\psi}_{1} \psi_{1} \bar{\psi}_{2} \psi_{2}$. Из всех этих инвариантных кривых только одна остается в области, ограниченной по $r$.

Пусть

$$
\begin{aligned}
g_{2}(r) & =\frac{\left(1-n^{1-\alpha}\right) r}{\left(1-n^{-\alpha}\right) r+1-n^{-1}}(1+r)^{2}, \\
G_{3} & =\left\{(r, g): r_{c}<r \leq 0, g_{2}(r) \leq g<0\right\},
\end{aligned}
$$

где $r_{c}=-\left(1-n^{-1}\right) /\left(1-n^{-\alpha}\right)$. Несложно проверить, что

$$
r^{\prime}(r, g)-r=\left(n^{\alpha}-1\right) \frac{r-r_{c}}{g-n(r+1)^{2}}\left(g-g_{2}(r)\right) .
$$

Отсюда следует лемма 10. 
ЛЕмма 10. Пусть $g<0$. Для всех точек $(r, g)$ таких, что $r<r_{c}$ или $(r, g) \in G_{3}$, имеет место неравенство $r^{\prime}(r, g)<r$. Для всех точек $\left(r, g_{2}(r)\right)$ верно $r^{\prime}(r, g)=r$. Для всех остальных точек нижней полуплоскости $r^{\prime}(r, g)>r$.

Очевидно также, что для всех точек нижней полуплоскости $g^{\prime}(r, g)<g$ при $\alpha \geq 3 / 2$.

Поскольку конечных НТ в нижней полуплоскости нет, $g$-координата РГ-итераций любой точки нижней полуплоскости стремится к $-\infty$. Если $R^{n}(r, g)$ асимптотически стремится к прямой $r=b$, где $b$ - некоторая константа, то из монотонного характера РГ-итераций следует, что $b$ должна удовлетворять уравнению

$$
b=n^{\alpha-1}(n(1+b)-1),
$$

т.е. $b=r_{c}$.

ТЕОрема 2. Существует функция $g=h_{3}(r), r_{c}<r \leq 0$, график которой является единственной неустойчивой (для тривиальной $Н T$ ) РГ-инвариантной кри вой, лежащей в области, ограниченной по $r$. Функиия $h_{3}(r)$ монотонно возрасmaem no $r, h_{3}(r) \rightarrow-\infty$ nрu $r \rightarrow r_{c}, h_{3}(0)=0, h_{3}(r)>g_{2}(r)$ npu $r_{c}<r<0$.

ДокАЗАТЕЛЬСтво. Перейдем к координатам $(r, t)$, где $t=g^{-1}$. В этих координатах РГ-преобразование запишется как

$$
\begin{aligned}
& r^{\prime}=n^{\alpha-1}\left(\frac{t(r+1)^{2}-1}{t(r+1)^{2}-1 / n}(r+1)-1\right), \\
& t^{\prime}=n^{3-2 \alpha}\left(\frac{t(r+1)^{2}-1 / n}{t(r+1)^{2}-1}\right)^{2} t
\end{aligned}
$$

Обозначим преобразование $(24),(25)$ через $\widetilde{R}: \widetilde{R}(r, t)=\left(r^{\prime}, t^{\prime}\right)$. Точка $\left(r_{c}, 0\right)$ является НТ $\widetilde{R}$. Собственные числа дифференциала отображения $\widetilde{R}$ в $\left(r_{c}, 0\right)$ равны $\mu_{1}=n^{\alpha}$ и $\mu_{2}=n^{1-2 \alpha}$, причем первому собственному числу отвечает собственньй вектор $e_{1}=(0,1)$, а второму - вектор $e_{2}=(1, a)$, где $a=-(n-1)^{-1}\left(1+r_{c}\right)^{-3}\left(1-n^{1-3 \alpha}\right)$.

Поскольку $0<\mu_{2}<1<\mu_{1}$, из обшей теории динамических систем (см., например, [7]) следует, что сушествует единственная устойчивая $\widetilde{R}$-инвариантная кривая для точки $\left(r_{c}, 0\right)$, причем в точке $\left(r_{c}, 0\right)$ эта кривая касается вектора $e_{2}$.

Легко проверить, что производная функции $t=g_{2}^{-1}(r)$ в точке $r_{c}$ больше, чем $a$. С другой стороны, $a<0$. Отсюда заключаем, что устойчивая $\widetilde{R}$-инвариантная кривая в окрестности точки $\left(r_{c}, 0\right)$ лежит в области $\widetilde{G}_{3}$, ограниченной слева прямой $r=r_{c}$ и справа кривой $t=g_{2}^{-1}(r)$. Применяя к этой части инвариантной кривой итерации отображения $\widetilde{R}^{-1}$, мы получим всю эту кривую. Из леммы 10 следует, что кривая целиком лежит в области $\widetilde{G}_{3}$ и является графиком монотонно убываюшей функции, стремящейся к $-\infty$ при $r \rightarrow 0$. Единственность этой кривой следует из теоремы о единственности устойчивой инвариантной кривой. Все остальные $\widetilde{R}$-инвариантные кривые являются неустойчивыми для точки $\left(r_{c}, 0\right)$ и касаются в ней прямой $g=0$.

Возврашаясь к координатам $(r, g)$, получаем утверждение теоремы. 
При $1<\alpha<3 / 2$ единственность РГ-инвариантной кривой, стремяшейся $\mathrm{k}-\infty$ по $g$ и ограниченной по $r$, остается в силе, но другим концом эта кривая входит уже в нетривиальную плюсовую НТ, которая при этих значениях $\alpha$ находится в нижней полуплоскости. Анализ спектра дифференциала РГ в плюсовой НТ показывает, что существует $\alpha_{0}<3 / 2$ такое, что при $\alpha<\alpha_{0}$ плюсовая НТ становится неустойчивым фокусом.

Компьютерный эксперимент показывает, что при $\alpha<\alpha_{0}$ устойчивые инвариантные кривые для тривиальной и бесконечно удаленной НТ “наматываются" на плюсовую НТ. При $\alpha_{0} \leq \alpha<3 / 2$ эти кривые выходят из плюсовой НТ. Таким образом, нижняя полуплоскость делится на две части: в левой части $r$-координата итераций РГ стремится к $-\infty$, в правой $-\mathrm{k}+\infty$.

Отметим, наконец, что гладкость всех перечисленных выше инвариантных кривых $\gamma_{1}, \gamma_{2}, \gamma_{3}$ следует из локальных теорем о гладкости инвариантных кривых в окрестности НТ.

Работа выполнена при поддержке Российского фонда фундаментальных исследований (код проекта 95-01-00270) и Международного научного фонда (код гранта RHA300).

\section{Список литературы}

[1] E. Yu. Lerner, M. D. Missarov. J. Stat. Phys. 1994. V. 76. № 3/4. P. 805.

[2] P. M. Bleher, Ya. G. Sinai. Commun. Math. Phys. 1973. V. 33. № 1. P. 23.

[3] Я. Г. Синай. Теория фазовых переходов. М.: Наука, 1980.

[4] P. Collet P., J.-P. Eckmann. A renormalization group analysis of the hierarchical model in statistical mechanics. Lecture Notes in Physics. V. 74. Berlin-Heidelberg-New York: Springer-Verlag, 1978.

[5] P. M. Bleher, P. Major. Ann. Prob. 1987. V. 15. P. 431.

[6] Э. Ю. Лернер, М. Д. Миссаров. ТМФ. 1996. Т. 107. № 2. С. 201.

[7] Ж. Палис, В.ди Мелу. Геометрическая теория динамических систем. М.: Мир, 1986.

Поступила в редакцию 22.V.1997 г., после переработки 8.XII.1997 г. 